\title{
The synthesis and characterization of novel brush-type chiral stationary phase based on terpenoid selector for resolution of chiral drugs
}

\author{
Dao-Cai Wang ${ }^{1}$, Jia Luo ${ }^{2}$, Shun Yao ${ }^{1}$ and Hang Song ${ }^{1, a}$ \\ ${ }^{1}$ College of Chemical Engineering, Sichuan University, 610065 Chengdu, China \\ ${ }^{2}$ Chengdu University of Traditional Chinese Medicine, 610075 Chengdu, China
}

\begin{abstract}
In the light of the chiral resolution mechanism and structures of brush-type CSP, a new chiral selector 4'-carboxyl-1'-ursolic methyl ester-3 $\beta$-yl-benzoate has been prepared. Then the terpenoid chiral selector was covalently linked to 3 -aminopropyl silica gel. Its structure identification data are provided by ${ }^{1} \mathrm{H}$ NMR, MS and elementary analysis. The enantiodiscriminating capability of the brush-type CSP was evaluated by static adsorption experiment with methyl mandelate, aniline derivative of mandelic acid, benzoin and ibuprofen. Experimental results demonstrated that the chiral selector has selectivity, and the enantiomers of methyl mandelate and ibuprofen could be separated on the CSP, which indicated that the novel brush-type CSP possess a bright prospects for chiral separation potentially.
\end{abstract}

\section{Introduction}

Molecular chirality is a fundamental property with evident implications on the mechanism of action at molecular level of bioactive compounds. Usually, the enantiomers of these bioactive molecules exhibit different physiological effects following different pathways in biological processes. The development of enantiopure drugs resulted in significant improvement in the remedies available for the treatment of various severe sicknesses [1]. As a result, the enantiomeric separation of chiral pharmaceuticals has become an increasingly interesting and important unit operation in the pharmaceutical industry [2]. Over the last three decades, great efforts has been made to isolate enantiomers, so that a large number of techniques has been employed for quickly obtaining limited quantities of pure single enantiomers, including diastereomer crystallization [3], membrane separation [4], chromatographic chiral separation $[5,6]$ and so on. Among the various approaches, liquid chromatography (LC) packed with chiral stationary phase (CSPs) has arosen increasing attention since its excellent enantioselectivities in a wide range of enantiomers. The chiral selector employed in the CSPs plays a key role in discriminating specific enantiomers, which is the central unit that relates to the chromatographic performance.

Since introduction to the area of chiral separation, the chiral selectors obtained from the natural chiral pool, such as alkaloids [7-10], sterols [11-13], terpenoids [14], and tartaric acid derivatives [15], have proved their usefulness and value in HPLC, which are used as the chiral molecular recognition site of brush-type CSPs. As a new class of adsorbents, brush-type CSPs can be easily and selectively modified allowing a prompt evaluation of the structural changes on the adsorption process. In an attempt to find other chiral selectors derived from these inexpensive natural raw materials with a well recognized high trade value, ursolic acid was selected as a terpenoid chiral selector of novel brush-type CSPs. Herein we report the synthesis, structural characterization, adsorption enantioselectivity of the novel brush-type CSP derived from ursolic acid.

\section{Experimental}

\subsection{Chemicals}

Silica gel (particle size, $7 \mu \mathrm{m}$ ) was purchased from Qingdao Haiyang Chemical Co., Ltd. (Qingdao, China). 3-(Trimethoxysilyl)-1-propanamine, iodomethane, terephthaloyl chloride, 4-dimethylaminopyridine and potassium carbonate were provided by Chengdu Kelong Chemical Co., Ltd. (Chengdu, China). Ursolic acid and EEDQ were acquired from Shanghai Hanhong Chemical Co., Ltd. (Shanghai, China). The racemic mandelic acid, benzoin and ibuprofen were produced by Sigma (USA). $\mathrm{n}$-Hexane and isopropanol (HPLC grade) were purchased from Chengdu Kelong Chemical Co., Ltd. (Chengdu, China). Other reagents were acquired from different commercial sources.

\subsection{Instruments and measurements}

\footnotetext{
${ }^{\mathrm{a}}$ Corresponding author: hangsong@scu.edu.cn
} 
The analysis of sample concentration was performed on a Shimadzu series liquid chromatography system, equipped with LC-20AT pump, SPD-M20A photodiode array detector (Kyoto, Japan), JASCO (Japan) model OR-2090 optical rotation detector, and HCT-360 LC column box (Tianjin, China). Chiralcel OD-H $(250 \times 4.6 \mathrm{~mm}$; particle size $5 \mu \mathrm{m})$ (Daicel, Japan), DNB-PG $(250 \times 4.6 \mathrm{~mm}$; particle size $5 \mu \mathrm{m})$, Whelk-O1 $(250 \times 4.6 \mathrm{~mm}$; particle size $5 \mu \mathrm{m}$ ) (Regis Technologies, USA) and Kromasil CHIDMB $(250 \times 4.6 \mathrm{~mm}$; particle size $5 \mu \mathrm{m})$ (Akzo Nobel, Sweden) were used for the separation of racemic samples.

\subsection{The synthesis of novel terpenoid chiral selector}

Ursolic acid ( $0.4 \mathrm{~g}, 0.88 \mathrm{mmol})$ was dissolved in acetone $(100 \mathrm{~mL})$ with the help of ultrasonic. In order to generate a carboxylic acid salt, potassium carbonate $(1.1 \mathrm{~g}, 8$ mmol) was added to the solution and stirred at room temperature until a milky solution was formed. Subsequently, iodomethane $(0.27 \mathrm{~mL}, 4.4 \mathrm{mmol})$ was dropped into the mixture, and stirred for another $4 \mathrm{~h}$. The solvent was evaporated under reduce pressure and the crude product was poured into water $(50 \mathrm{~mL})$ to wash the salt out of the mixture. And then the product was extracted with ethyl acetate. Further purification of ursolic methyl ester was accomplished by recrystallization with ethanol.

Next, a solution of ursolic methyl ester $(0.28 \mathrm{~g}, 0.6$ mmol $)$, terephthaloyl chloride $(0.61 \mathrm{~g}, 3 \mathrm{mmol})$ and 4 dimethylaminopyridine $(0.12 \mathrm{~g}, 1 \mathrm{mmol})$ in dry toluene $(25 \mathrm{~mL})$ was heated to reflux for $8 \mathrm{~h}$. The toluene was removed by distillation in vacuo and the gained crude product was dissolved in THF $(60 \mathrm{~mL})$. The $\mathrm{pH}$ value of the solution was adjusted to 9 by adding $5 \%$ sodium carbonate solution, and then the $\mathrm{pH}$ value was adjusted to 3 by adding hydrochloric acid. The mixture was extracted with dichloromethane and the organic layer was obtained by the centrifugal separation of liquid and solid. The organic solvent was removed on a rotary evaporator in vacuo to give a crude product of $4^{\prime}$-carboxyl-1'-ursolic methyl ester-3 $\beta$-yl-benzoate, which was subjected to silica gel column separation eluting with dichloromethane/acetic acid (100/1, v/v). This new terpenoid chiral selector was white solid with a yield of $82 \%$, which was characterized by HPLC, ${ }^{1} \mathrm{H}$ NMR and MS. mp.298 $299^{\circ} \mathrm{C},{ }^{1} \mathrm{H}$ NMR $\delta: 8.14-8.21$ (dd, $4 \mathrm{H}, \mathrm{J}=$ $8 \mathrm{~Hz}), 5.28-5.32(\mathrm{~m}, 1 \mathrm{H}), 4.77-4.81(\mathrm{~m}, 1 \mathrm{H}), 3.64(\mathrm{~s}, 3 \mathrm{H})$, $0.79-2.28(\mathrm{~m}, 45 \mathrm{H}) ; \mathrm{MS}(\mathrm{ESI}): \mathrm{m} / \mathrm{z}=619.63[\mathrm{M}+\mathrm{H}]^{+}$.

\subsection{Preparation of brush-type chiral stationary phase}

Dried silica gel (5 g) and 3-(trimethoxysilyl)-1propanamine $(6 \mathrm{~g})$ were added in dry toluene $(50 \mathrm{~mL})$. The resulting mixture was heated to reflux, and stirred for $36 \mathrm{~h}$ under a nitrogen atmosphere. After the completion of the reaction, the solid was filtered and washed successively with toluene, methanol, diethyl ether and npentane. The obtained 3-aminopropyl silica gel was dried in vacuum oven overnight at $60^{\circ} \mathrm{C}$.
Dry 3-aminopropyl silica gel (2.3 g) was suspended in the solution of the above freshly prepared chiral selector $(2.2 \mathrm{~g})$, EEDQ $(1.8 \mathrm{~g})$ in dried THF $(65 \mathrm{~mL})$. The suspension was gently stirred at room temperature for 12 $\mathrm{h}$ under a nitrogen atmosphere. After the completion of the amidation reaction, the solid was collected by filtration and was washed, respectively, with THF, acetone, n-hexane and diethyl ether to afford the brushtype CSP, which was dried in vacuo overnight.

\subsection{Static adsorption of four samples on the adsorbent}

The equilibrium adsorption of the absorbent was determined with static method. In the procedure, $0.5 \mathrm{~g}$ of absorbent was placed into $50 \mathrm{~mL}$ conical flasks and mixed with $20 \mathrm{~mL}$ organic solution of four chiral samples with different concentrations $\left(C_{0}\right)\left(0.1-1.0 \mathrm{mg} \mathrm{mL} \mathrm{m}^{-1}\right)$, respectively. Then the suspension was stirred by a magnetic stirrer at the temperature of $30^{\circ} \mathrm{C}$ for $24 \mathrm{~h}$ to ensure adsorption equilibrium. Finally, the suspensions were centrifuged and two enantiomer concentrations of each chiral sample $\left(C_{\mathrm{e}}\right)$ in supernatant were detected by HPLC. The equilibrium adsorption capacity $\left(Q_{\mathrm{e}}, \mathrm{mg} \mathrm{g}^{-1}\right)$ of each enantiomer on the adsorbent was calculated according to the eq. (1):

$$
Q_{\mathrm{e}}=\frac{V\left(C_{0}-C_{\mathrm{e}}\right)}{m}
$$

In addition, to illustrate the practical value of the adsorbent in chiral separation, the enantiomeric excess (e.e.\%) of the chiral sample solution was calculated by the following equation:

$$
\mathrm{ee} \%=\frac{[\mathrm{S}]-[\mathrm{R}]}{[\mathrm{S}]+[\mathrm{R}]} \times 100 \%
$$

where $[R]$ and $[S]$ are the concentrations of the Renantiomer and $\mathrm{S}$-enantiomer in the solution.

\section{Results and discussion}

\subsection{Preparation and Characterization of CSP}

The preparing process of the novel brush-type CSP derived from ursolic acid was as follows (Figure 1). As a pentacyclic triterpene acid existing abundantly in the plant kingdom, ursolic acid is a promising chiral selector with multiple chiral centers. According to the "three point principle" theory in the design of new CSP, considerable structural modifications are looking forward to obtaining a potential chiral selector with enhanced physical/chemical properties, so that they are capable of most effectively activating donor-acceptor interactions such as hydrogen bonding, face-to-face or face-to-edge $\pi$ $\pi$ interaction, and dipole-dipole-stacking. The most convenient modifications of ursolic acid are obviously on the alcohol group at position C3 and on the carboxylic acid group at position $\mathrm{C} 28$ with the form of new amides or esters. To reduce the production of by-products, the esterification of 28-carboxyl group with iodomethane was 
performed previously. Then the 3-hydroxyl group was reacted with paraphthaloyl chloride to introduce the benzene ring, which acted as an important $\pi-\pi$ interaction group. After the hydrolysis of the acyl chloride, the obtained chiral selector was bonded to 3-aminopropyl silica gel with the generation of amide.
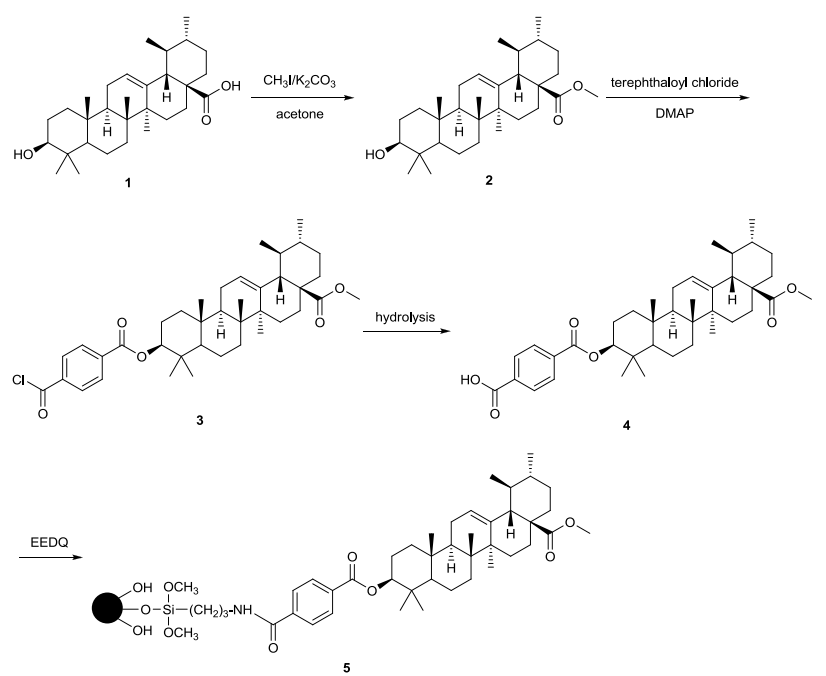

Figure 1. Synthesis process of new brush-type chiral stationary phase.

In order to calculate the selector loading, a sample was sent for elemental analysis. Elemental analysis of 3aminopropyl silica gel (C, 2.66; H, 1.46; N, 1.38) showed a loading of $0.985 \mathrm{mmol} / \mathrm{g}$ of 3-(trimethoxysilyl)-1propanamine (based on $\mathrm{N}$ ), and elemental analysis of the brush-type CSP derived from ursolic acid (C, 10.18; H, $2.18 ; \mathrm{N}, 1.38$ ) showed a loading of $0.161 \mathrm{mmol} / \mathrm{g}$ of the chiral selector (based on $\mathrm{C}$ ). The selector loadings are calculated according to the following formula:

$$
\text { Selector loading }=10 \times \frac{\Delta C}{M \times n} \mathrm{mmol} / \mathrm{g}
$$

in which $\Delta C$ represents the content increment of carbon or nitrogen; $M$ is the relative atom mass of carbon or nitrogen; $n$ is the number of the atom (carbon or nitrogen) contained in 3-(trimethoxysilyl)-1-propanamine molecule or chiral selector molecule.

\subsection{The HPLC analysis of the four chiral samples}

Four chiral samples were selected as the target analytes in the experiment (Figure 2), and four different commercially available chiral columns were employed for the chiral separation as follows: Chiralcel OD-H, DNB-PG, Whelk-O1 and Kromasil CHI-DMB. The chiral separation of racemate (a) was performed on Kromasil CHI-DMB using n-hexane: isopropanol (98:2, $\mathrm{v} / \mathrm{v}$ ) as mobile phase with a flow rate of $0.6 \mathrm{~mL} \mathrm{~min}^{-1}$ at $25^{\circ} \mathrm{C}$. Similar conditions were used to the chiral separation of racemate (b), only the mobile phase was replaced by $n$-hexane: isopropanol $(90: 10, \mathrm{v} / \mathrm{v})$. Chiralcel OD-H was employed for the chiral separation of racemate (c) using n-hexane: isopropanol $(90: 10, \mathrm{v} / \mathrm{v})$ as mobile phase, and Whelk-O1 was used for the enantiomeric separation of racemate (d) using n-hexane: isopropanol $(98.5: 1.5, \mathrm{v} / \mathrm{v})$ as mobile phase with other conditions unchanged. To find out the relationship between instrumental response and known concentrations of pure sample solution, calibration curves were constructed with high linearity.
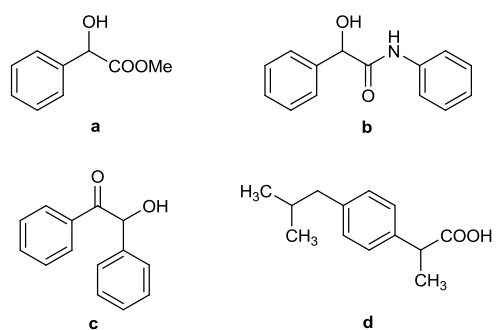

Figure 2. The structures of four racemates (a-d) used in the adsorption.

\subsection{Adsorption studies}

The adsorption isotherms of the adsorbent for the four racemates at $30^{\circ} \mathrm{C}$ are shown in Figure 3 . It can be concluded that methyl L-mandelate, aniline derivative of L-mandelic acid, (S)-(+)-benzoin and (R)-()-ibuprofen are more easily adsorbed to the adsorbent with a higher equilibrium adsorption capacity than the other enantiomer.
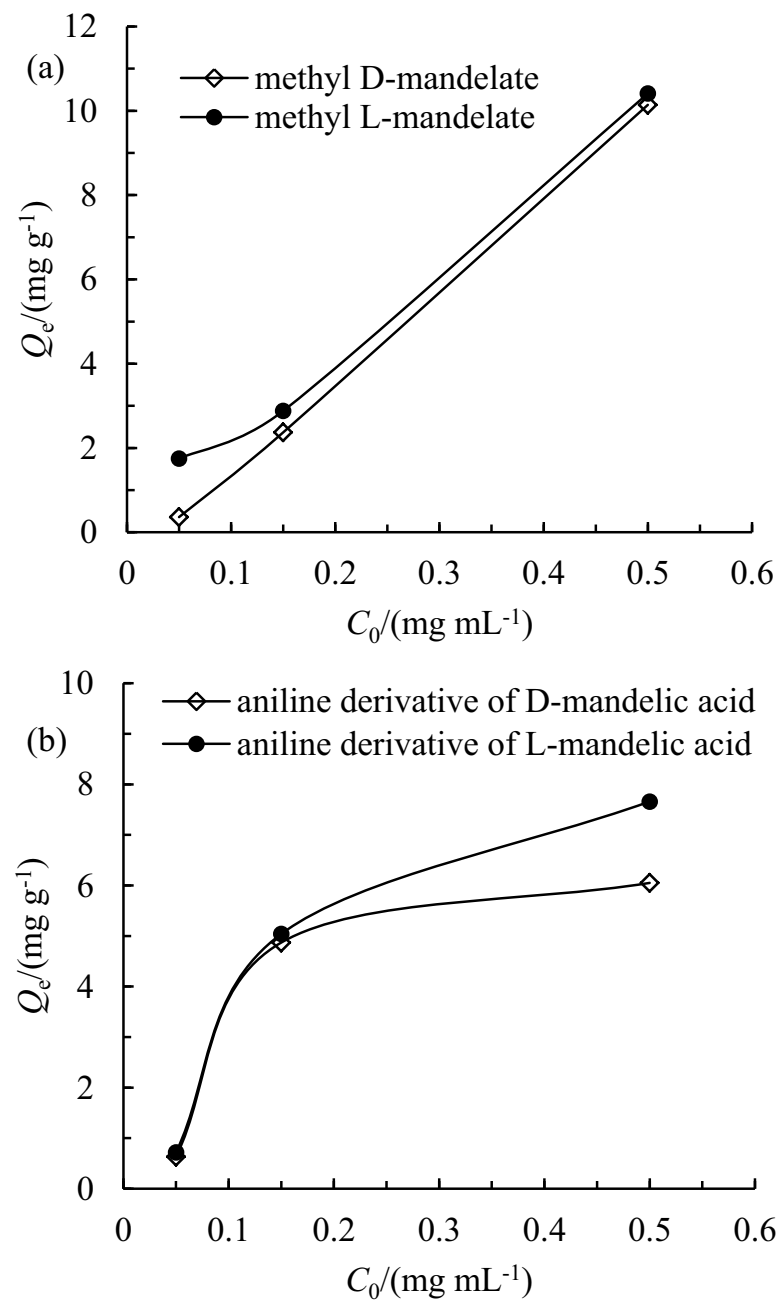

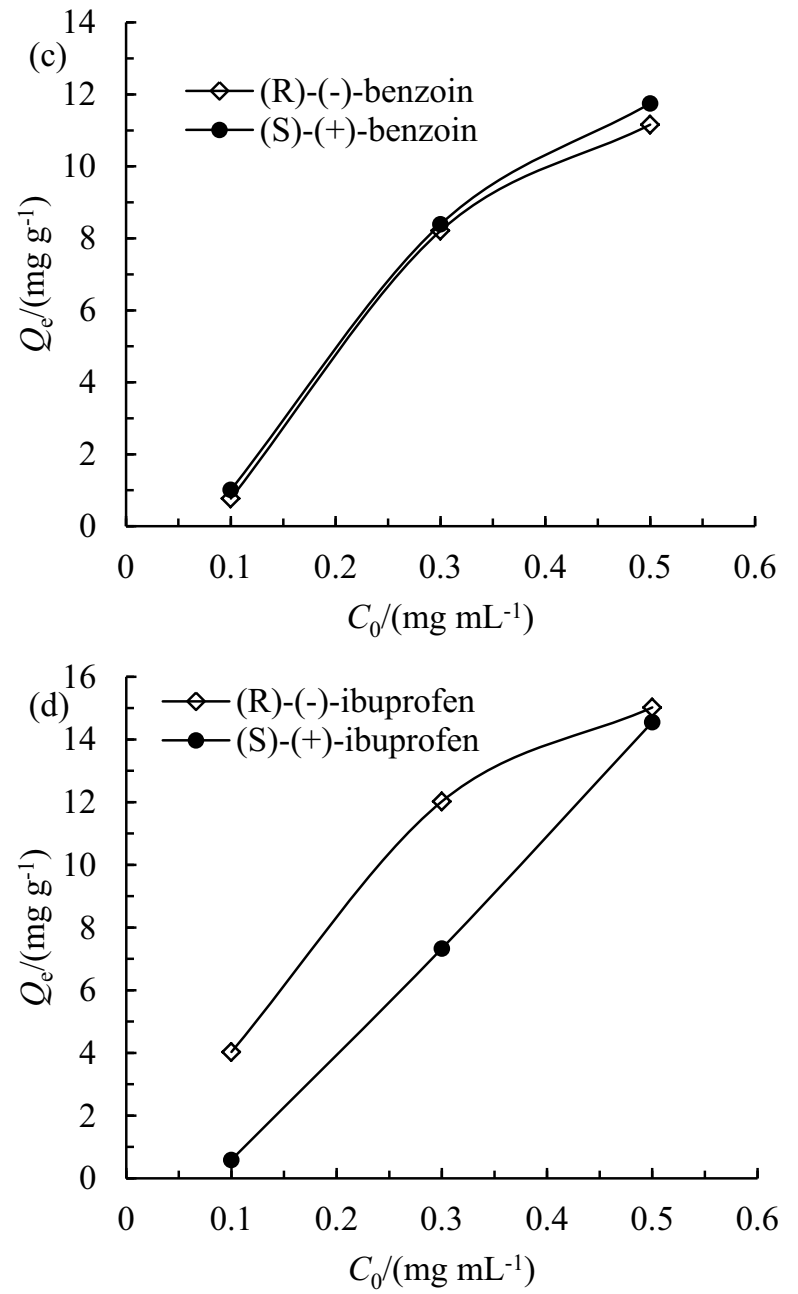

Figure 3. The equilibrium adsorption capacity of the absorbent for the four racemates.

The selectivity of adsorption to the enantiomers (e.e.\%) is given in Figure 4, which reveal that the enantiomers of methyl mandelate and ibuprofen could be separated on the adsorbent to some extent especially at lower concentrations. As expected, the novel brush-type CSP could be used as potential adsorbent to achieve the resolution of chiral pharmaceuticals.

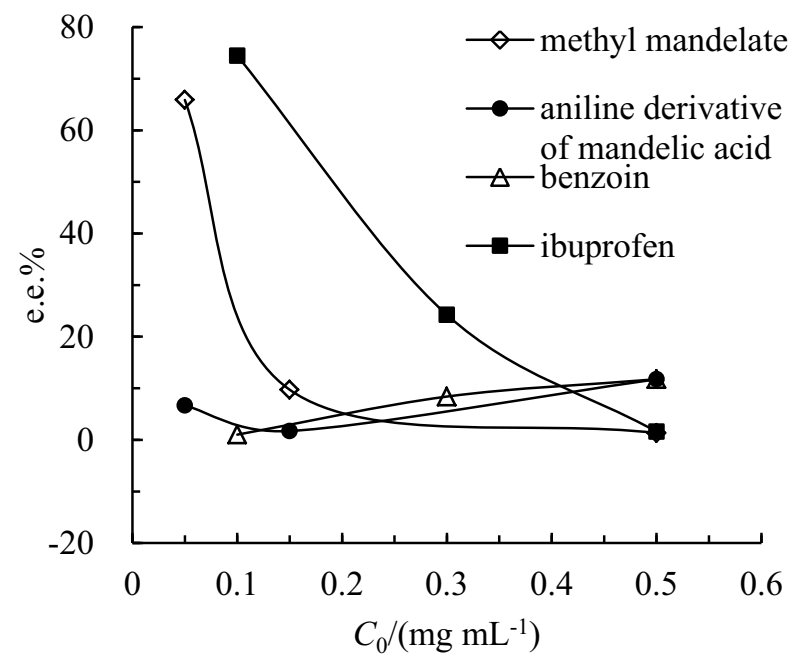

Figure 4. The e.e. $\%$ of four racemates after adsorption on the absorbent.

\section{Conclusions}

According to the chiral resolution mechanism and structures of brush-type CSP, a new chiral selector 4'carboxyl-1'-ursolic methyl ester-3 $\beta$-yl-benzoate has been prepared in this work. The terpenoid chiral selector has been covalently linked to 3 -aminopropyl silica gel. Then the enantiodiscriminating capability of the brush-type CSP was evaluated by static adsorption experiment with methyl mandelate, aniline derivative of mandelic acid, benzoin and ibuprofen. Experimental results demonstrated that the chiral selector has selectivity, and the enantiomers of methyl mandelate and ibuprofen could be separated on the CSP to some extent especially at lower concentrations. As been seen that, the large retention capacity and good selectivity to the enantiomers made the novel brush-type CSP possess a bright prospects for chiral separation potentially.

\section{References}

1. B. S. Sekhon, J. Mod. Med. Chem. 1, 10 (2013)

2. O. Mcconnell, A. Bach, C. Balibar, N. Byrne, Y.X. Cai, G. Carter, M. Chlenov, L. Di, K. Fan, I. Goljer, Y.N. He, D. Herold, M. Kagan, E. Kerns, F. Koehn, C. Kraml, V. Marathias, B. Marquez, L. McDonald, L. Nogle, C. Petucci, G. Schlingmann, G. Tawa, M. Tischler, R.T. Williamson, A. Sutherland, W. Watts, M. Young, M.Y. Zhang, Y.R. Zhang, D.H. Zhou, D. Ho, Chirality 19, 658 (2007)

3. C.R. Baley, V.A. Vaidya, Resolution of Racemates by Diastereomeric Salt Formation CR BayleyChirality in Industry. New York: John Wiley \& Sons, 1992

4. W.H. Pirkle, W.E. Bowen, Tetrahedron Asymmetry 5, 773 (1994)

5. C.V. Gonalves, M.J.S. Carpes, C.R.D. Correa, C.C. Santana, Chem. Eng. J. 133, 151 (2007)

6. K.L. Sutton, C.A.P. de Leon, K.L. Ackley, R.M.C. Sutton, A.M. Stalcup, J.A. Caruso, Analyst 125, 281 (2000)

7. F. Gasparrini, D. Misiti, C. Villani, J. Chromatogr. A 906, 35 (2001)

8. A. Mandl, L. Nicoletti, M. Lämmerhofer, W. Lindner, J. Chromatogr. A 858, 1 (1999)

9. K.H. Krawinkler, N.M. Maier, E. Sajovic, W. Lindner, J. Chromatogr. A 1053, 119 (2004)

10. P. Franco, M. Lämmerhofer, P.M. Klaus, W. Lindner, J. Chromatogr. A 869, 111 (2000)

11. A. Iuliano, P. Salvadori, G. Félix, Tetrahedron: Asymmetry 10, 3353 (1999)

12. A. Iuliano, G. Félix, J. Chromatogr. A 1031, 187 (2004)

13. A. Iuliano, A. Ruffini, Tetrahedron: Asymmetry 16, 3820 (2005)

14. C. Moiteiro, N. Fonseca, M.J.M. Curto, R. Tavares, A.M. Lobo, P. Ribeiro-Claro, V. Félix, M.G.B. Drew, Tetrahedron: Asymmetry 17, 3248 (2006)

15. Y. Machida, H. Nishi, K. Nakamura, H. Nakai, T. Sato, J. Chromatogr. A 757, 73 (1997) 